\title{
Supporting Nursing Leadership by the Implementation of an increased Supervisory Role
}

\author{
Jean Rankin , Clare McGuire, Lynsay Matthews, Margot Russell, and Devashish Ray
}

\begin{abstract}
Clinical nurse leadership is essential for the provision of high quality patient care. Recent research has highlighted the need for all Senior Charge Nurses (SCNs) to be equipped to deliver effective ward management, staff development and ultimately a positive patient experience. The provision of 'supervisory hours' is a vital component of an SCN role, enabling the effective leadership of their teams. This paper describes the increased supervisory role implemented by the Leading Better Care program within one Health Board area of Scotland. Mixed methods research (online survey $n=52$ and in-depth qualitative interviews $n=12$ ) explored the experiences of SCNs using their increased supervisory time and its perceived impact on clinical practice. Findings demonstrate that increased supervisory time positively impacted several key areas of clinical practice. Although the increased supervisory role empowered SCNs and promoted their professional development, they require greater role clarity, managerial support and leadership development to reduce role stress and promote future career aspirations.
\end{abstract}

Keywords-Nursing Leadership; Supervisory Role; Senior Nurses; Workforce Planning.

\section{INTRODUCTION}

Clinical nurse leadership is a cornerstone of high quality patient care. It is recognized as being vital for effective ward management, staff development and a positive patient experience [1]. Numerous studies have explored the role of clinical leadership with findings suggesting that senior nurses often do not have the necessary leadership qualities and resources to effectively manage clinical teams [2-4]. This was highlighted in critical reports, such as the Willis Commission and Francis Report [5, 6] whose findings identified the need for senior nurses to be appropriately equipped for leadership and delivery high quality patient care.

The Scottish Government define and support leadership in Nursing and Midwifery through the policy, Leading Better Care (LBC). LBC provides a framework to provide role clarity for senior nurses in Scotland. The framework aims to: (i) ensure safe and effective clinical practice; (ii) manage and develop the performance of the

This study was funded by the Leading Better Care program, a collaborative initiative between NHS Lanarkshire and University of the West of Scotland. team; (iii) enhance the patient experience; and (iv) ensure effective contribution to the organization's objectives [7]. In particular, the role of senior nurses (known as Senior Charge Nurses [SCNs] in Scotland) is considered pivotal in achieving these aims. Research has shown that the role of SCNs has changed significantly in recent years, with greater responsibility and emphasis for duties out with patient care [8]. For example, SCNs manage duties such as clinical audits, ward budgets and staff rosters. Additional responsibilities include managing compliance with professional regulatory rules, organizational policies and procedures, and quality assurance systems [8]. It was recognized that due to this significant change in responsibility SCNs required support to develop their leadership skills and empower them as clinical leaders.

Recent research highlighted the benefits to SCNs of increasing their supervisory role and reducing their patient caseload $[9,10]$. A key issue in supporting leadership was, therefore, to address the balance between time spent with a specified patient caseload and time spent supervising and leading their clinical teams.

One Health Board in Scotland (NHS Lanarkshire) took this guidance on board and implemented an increased supervisory role for SCNs [10]. In 2013 SCNs from acute hospital settings in NHS Lanarkshire were provided with 22.5-hours per week of supervisory time, an increase from their previous 7.5-hours per week allocation. The purpose of the designated time was to provide effective leadership, supervision and management of the ward team, with the overall aim of ensuring the delivery of safe, effective and person centered care.

Recent evidence suggests that from the perspective of the SCN, LBC has the potential to improve (i) the effectiveness of their supervisory role, (ii) performance of the nursing team, and (iii) overall care delivery for patients [8].

This study explores the experiences of those SCNs who were provided with an increased supervisory role. The specific aim of the study was to identify the perceived impact of the increased supervisory role on leadership practice. 


\section{METHODS}

A mixed methods approach, conducted over two phases, was used to explore the impact of SCNs increased supervisory role on practice.

\section{A. Phase One}

Phase One involved a survey questionnaire design to gather data from SCNs based in three acute hospitals within one Health Board area in Scotland $(n=60)$. The online questionnaire was designed to collect: (i) SCN demographics; (ii) explore the experiences of SCNs working in an increased supervisory role; and (iii) explore how SCNs used their increased supervisory time to address the four key areas of LBC. The questionnaire consisted of 38 questions; twelve of which used a four point Likert scale to gather quantifiable data. The remaining questions gathered descriptive responses that would be analyzed to present qualitative data. An online web link was emailed to SCNs for participation. Responses were anonymously collected by a secure online server.

\section{B. Phase Two}

Phase Two used a qualitative design to gather indepth insight from a smaller sample of SCNs $(n=12)$. One-to-one interviews were conducted with a University researcher. The interview schedule was semi-structured, informed by key issues raised in Phase One. This approach allowed a degree of flexibility and enabled participant responses to be explored. In general, SCNs were asked to reflect on: (i) a typical working day; (ii) factors that shape their work environment; and (iii) the impact of their increased supervisory role upon patient care, the patient experience, their personal development and the overall development of their ward team. Interviews were transcribed verbatim and thematically analyzed for meaningful data. Findings were discussed within the research team and categorized into themes and sub-themes.

\section{Data analysis}

Numerical data from Phase One were summarized as frequencies and percentages, with selected data presented in graphical format. Qualitative data from both Phase One and Phase Two were transcribed verbatim, analyzed and discussed within the research team to identify common themes related to the impact of supervisory hours [11].

\section{Ethical Approval}

Access to participants for both phases of the study was granted by NHS Lanarkshire's Research and Development department. Ethical approval was granted from the University of the West of Scotland's ethics committee. Standard ethical procedures were adhered to using a confidential survey site, providing participant information, gaining consent and protecting anonymity of participants. The Research and Evaluation subgroup of the LBC program informed and guided the research design and supported the implementation of the study.

\section{FINDINGS}

\section{A. PHASE ONE: Findings from the online questionnaire.}

The online survey received a high response rate of $87 \%$ ( $n=52$ of 60 SCNs). The majority of SCNs had been a SCN for less than 10 -years $(58 \%, n=30$ of 52$)$ and had been working in an increased supervisory role for less than 20 -weeks $(60 \%, n=31$ of 52$)$. Although allocated 22.5 -hours of supervisory time per week, $81 \%(n=42$ of 52 ) were only managing to use 15 -hours or less per week on average in the preceding month.

In general, SCNs responded positively when asked to reflect on the impact of increased supervisory time on practice (Fig. 1-2).

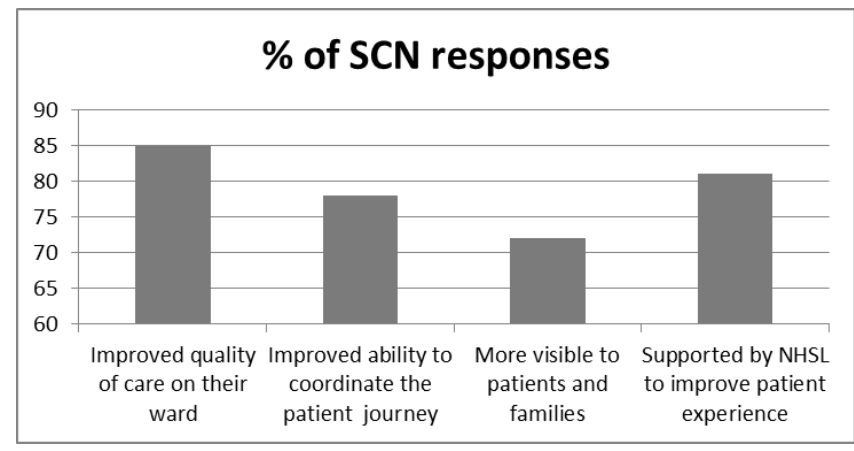

Fig. 1. Responses from SCNs when asked about their perception of how increased supervisory time impacted on the patient experience.

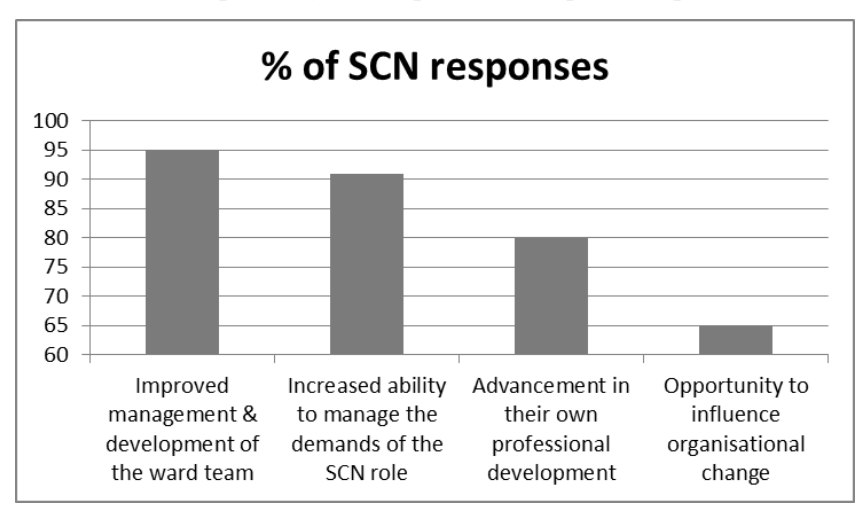

Fig. 2. Responses from SCNs when asked about their perception of how increased supervisory time impacted on their professional development and development of their staff. 


\section{B. PHASE TWO: Findings from the semi-structured interviews.}

Two key themes were identified: Impact of increased supervisory hours on practice; and impact of increased supervisory role on the SCN.

Theme 1. Impact of increased supervisory hours on practice

SCN's identified four areas which reflected the impact of the increased supervisory role on practice. These included: (i) improved quality of patient care and patient safety; (ii) improved compliance with audits and documentation; (iii) positive patient and family experience; and (iv) development of the ward team.

\section{- Improved quality of care and patient safety}

SCNs passionately expressed that their goal was to provide high quality patient care and safety. Their increased supervisory time enabled them to work towards this goal by releasing them from a full-time patient caseload.

'[Increased supervisory time has] allowed us to be innovative, it has allowed me to make changes. It has allowed us to look at the patient experience.'

Increased supervisory time was used in a variety of ways to improve the overall patient experience. Some SCNs described how they used the supervisory role to spend time on the ward and evaluate what changes or improvements were required. This enabled the development of new protocols that streamlined ward processes. At the time of this study, the majority of SCNs struggled to implement and embed these new ideas due to being unable to fully utilize their supervisory time.

- Improved compliance with audits, reports and documentation.

Increased supervisory time enabled SCNs to maintain clinical audits, quality indicators and other clinical requirements. When full supervisory time was available, SCNs were able to analyze the findings and take appropriate action. The collection of data which measured quality and safety of patient care allowed the evaluation of positive and negative trends on their ward. This enabled SCNs to provide timely feedback to staff, boosting staff morale, in addition to engaging staff in areas which required further improvement.

'I don't believe that the audits are a 'tick box exercise'. I believe from a perspective that we have factual [information] - whether we are doing a good job or where there're areas that have to be improved upon.'
SCNs perceived that the workload for completion of clinical audits and documentation had increased significantly. However, overall, they felt that standards of clinical documentation had improved. In the absence of staff shortage, some SCNs were able to delegate specific audit and report tasks to appropriately trained staff. This had the positive affect of harnessing support from staff and promoting staff development.

\section{- Positive patient and family experience.}

Increased supervisory time enabled SCNs to be a visible presence in their clinical areas. Being present on the ward without a defined patient caseload provided SCNs the opportunity to observe the ward processes in action. In particular, SCNs used this time to develop good communication with patients and families. SCNs believed this visible presence and open communication reduced both formal and informal complaints. They acknowledged this as an important outcome, which not only reflected high quality care, but also boosted staff morale.

$$
\begin{gathered}
\text { 'It has reduced the complaints... Because you are } \\
\text { dealing with them at the time. ...If they've got any } \\
\text { issues - they can approach it - you can deal with } \\
\text { it before they escalate.' }
\end{gathered}
$$

\section{- Development of the ward team}

Leading a competent and confident team was a key priority for SCNs. Supervisory time was used to build close working relationships with staff and support them with continuing professional development. They were aware of their responsibility to staff in relation to being a role model, mentor and supervisor. This aspect of their role was a source of satisfaction and pride for them.

\section{'I have a closer relationship with my staff because}

I am now afforded the time now to actually go out

there and be on the floor on a more regular basis.'

SCNs used their supervisory time to release staff, which supported attendance at training and development workshops. They also acted as an advocate for their staff, collating their concerns about patient safety, in particular staff shortages, and raising these concerns with managers. Advocacy, in conjunction with positive feedback, improved staff morale.

\section{Theme 2. Impact of increased supervisory hours on practice}

Three key factors were identified which reflected the impact of the increased supervisory role on the SCN: (i) 
empowerment and personal development; (ii) role stress; and (iii) modified career aspirations.

\section{- Empowerment and Personal Development}

Increased supervisory time has had a significant impact on the SCN. For newly appointed SCNs, who came into the post with increased supervisory hours as a component of their role, there has been time to; reflect upon the challenges of the role; identify immediate and long term goals and consider options for moving forward. This time has therefore supported an improved understanding of the role and identified strategies to achieve their goals.

\section{'I just think I was fortunate that I came}

in with the supervisory hours ... I think a lot of

SCNs that have been in the role for years find it hard to put it in their plan...'

SCNs enjoyed having responsibility for taking action and delivering effective changes with the supervisory role also empowering ownership of the role and of the ward. SCNs perceived that their position gave some influence over policy and practice through acting as an advocate for patients, families and staff. Most SCNs expressed that in spite of the challenges and work-related stress, they valued their increased supervisory role. They took pride in being an SCN and seeing the positive impact their work had on patients, families and staff.

'That's where you get satisfaction from, that's

my moments, when I see a patient getting out and

$$
\text { they're much better.' }
$$

\section{- $\quad$ Role Stress}

All SCNs in this study described the multidimensional aspect of their role as demanding and stressful. The focus on quality of care and safety was welcomed; however, it was challenging to manage the increased workload of administrative tasks and audits. Additional leadership responsibilities were therefore afforded less time.

SCNs reported that high bed occupancy sometimes led to pressure to discharge patients to ensure beds were available for the next patient. This pressure, in addition to the demands of managing the budget, ward resources and a team, made the job seem extremely challenging for SCNs.

Role stress was highlighted by SCNs in the study. The demands of the role often exceeded the resources available to SCNs, particularly in relation to available time and staff numbers. As such, prioritizing clinical leadership proved difficult leading to feelings of being overwhelmed by the demands of the job.
'Demoralized, undervalued, overworked,

$$
\text { stressed beyond belief.' }
$$

Demands on the SCNs time from a wide range of sources could lead to role overload. High expectations challenged the SCNs, who felt they were asked to do too many things, for too many people, in too little time. The perception of SCNs was that others - staff, patients, families and the organization - viewed them as responsible for everything.

Managing their workload, particularly when unable to utilize supervisory time, led to SCNs working longer hours and working at home SCNs were aware of the negative impact this could have on their personal life. A sense of growing disempowerment over their day to day work plan was reported by some SCNs. The changes increased supervisory time had brought was welcomed, however, over time they felt they were gradually losing the autonomy that was required to effectively carry out duties specific to the needs of their ward.

'It is still supervisory - but I feel I have lost a

wee bit control of that - in determining what I deem to be required within my ward.'

\section{- Aspirations of the SCN}

SCNs greatly valued their central role as the person with responsibility for patient, families and staff in their clinical area. Direct contact with patients and families was integral to their identity as a Registered Nurse. They passionately described how important dealing with patients was to their sense of purpose. They struggled during times of high workload, when they felt they were required to deal predominately with managerial administration. Although SCNs welcomed opportunities to influence patient care they perceived that their role was often focused on targets, audits and statistics. This caused personal conflict with their career aspirations. They emphatically stated that they did not want a role in nursing that would take them away from the patient contact that they deeply valued. They perceived more senior positions as those types of roles.

'I don't want to be in a senior management role.

I like patient contact and I don't want not to have

$$
\text { patient contact.' }
$$

Some SCNs also reported a future problem with junior staff aspiring to the role of SCN. They suggested that junior staff would be more motivated to undertake an SCN post if they could see that present staff were well prepared, developed and supported for the role. 


\section{DISCUSSION}

SCNs perform a complex and demanding role. Despite the challenges all SCNs in this study found the role rewarding and deeply valued their responsibility for patient care. Overall, findings demonstrated a positive impact of increased supervisory time on practice, with a mixture of positive and negative effects on the SCN. Firstly, the increased supervisory role positively impacted perceived practice via: (i) improved quality of patient care and safety; (ii) improved compliance with audits and clinical documentation; (iii) a positive patient and family experience; and (iv) greater development of the ward team. In order for SCNs to effectively lead their ward they require time to be visible to patients, staff and multidisciplinary colleagues [12]. Importantly these positive changes were observed within a short space of time. The majority of SCNs had been in an increased supervisory role for less than 5-months. It is therefore encouraging to have SCNs report a positive impact on four key areas within a short time frame. This suggests that SCNs, when provided with appropriate time and resources, have the ability to lead positive change within their ward.

Secondly, increased supervisory time positively impacted SCNs through empowerment and personal development; however, this was alongside significant role stress and modified career aspirations. Concerns were raised over the succession of junior nurses into senior nursing roles due to the perceived lack of support, role clarity and role stress of current SCNs. The reduced patient contact associated with the supervisory role highlighted to SCNs the deep value they placed in providing patient care. This appeared to reduce their motivation to pursue further management roles that would take them further away from nursing practice.

Role stress may be associated with 'barriers' to the supervisory role. Previous findings showed that SCNs experienced two key barriers to their development of ward leadership: (i) limited availability of supervisory time; and (ii) perceived lack of support for the role [13]. In the instance of staff shortage SCNs were required to manage a case load of patients, limiting their availability of supervisory time. A significant amount of time was often spent managing staff rosters and balancing a safe skill mix of permanent and temporary bank staff. The Royal College of Nursing (RCN), however, highlighted the importance of SCNs being acknowledged as 'supervisory' and not 'supernumerary' [14]. This issue was acknowledged in a business case by the RCN to demonstrate the cost-effectiveness of SCNs operating exclusively in a full-time supervisory role. This change in policy would, however, require additional investment to initially address staffing levels, in particular for SCNs who often need to manage additional patient caseloads $[15,16]$.

Previous studies have also shown that inadequate support from management acts as a barrier for SCNs to effectively perform their leadership role [9, 17]. Despite having confidence in their skills SCNs suggested further support to develop their leadership capacity [13]. Education, training and structural support play a critical role in the development of leadership and managerial skills [18-20]. Empowered SCNs who feel supported by their organization are more committed to the role and more likely to be influential role models for their teams [21].

In conclusion, supervisory time is essential to support and facilitate effective clinical leadership in Nursing and Midwifery. Consideration should be given to the allocation of full-time supervisory hours to enable SCNs to reach their leadership potential. Although the increased supervisory role empowered SCNs and promoted their professional development, they require greater role clarity, managerial support and leadership development to reduce role stress and promote future career aspirations. An increased supervisory role was perceived by SCNs to impact positively on several key areas. Further research is now required to explore the impact of the supervisory role on the overall patient experience.

\section{ACKNOWLEDGMENT}

The research team would like to thank: NHS Lanarkshire Research and Development department; the Leading Better Care Program Board, and Research and Evaluation group; and SCNs who participated in the study.

\section{REFERENCES}

[1] M. Douglas, "Opportunities and challenges facing the future global nursing and midwifery workforce," Journal of Nursing Management, vol.19, pp. 695-699, 2011.

[2] K. Fenton, and N. Phillips, "Developing skills in clinical leadership for ward sisters," Nursing Times, vol. 109(9), pp. 12 15.

[3] C. Agnew, and R. Flin, "Senior charge nurses' leadership behaviours in relation to hospital ward safety: A mixed method study," International Journal of Nursing Studies, vol. 51(5), pp. 768-780, 2014

[4] S. Allder, K. Silvester, and P. Walley, "Managing capacity and demand across the patient journey," Clinical Medicine, vol. 10(1), pp. 13-15, 2010. 
[5] R. Francis R, "Report of the Mid Staffordshire NHS Foundation Trust Public Inquiry -Volume 3: Present and future," The Stationery Office, London, 2013.

[6] Kings Fund, "Patient-centred Leadership: rediscovering our purpose," Kings Fund, London, 2013.

[7] C. McGuire, and D. Ray, "developing leadership roles in nursing and midwifery," Nursing Standard, vol. 29(9), pp. 43-49.

[8] K. Stoddart, C. Bugge, A. Shepherd, and B. Farquharson, "The new clinical leadership role of senior charge nurses," Journal of Nursing Management, E-pub DOI: 10.1111/jonm.12008, 2012.

[9] S. Cayless, C. Malcolm, and N.Kearney. "Patient and public perspectives of the role of the Ward Sister and Senior Charge Nurse: A review of the literature," Scottish Executive Health Department, Edinburgh, 2006.

[10] M. Russell, and C. McGuire, "Leading Better Care: Implementing supervisory status for Senior Charge Nurses, a description of two projects,” Nursing Standard, vol 29(12), pp. 37-43, 2014.

[11] V. Bruan, and V. Clarke, "Using thematic analysis in psychology," Qualitative Research in Psychology, vol. 3, pp. 77 101, 2006.

[12] Royal College of Nursing, "Staffing Key to PM's Quality Drive, Says RCN." Retrieved from: http://www.rcn.org.uk/newsevents/news/article/uk/staffing_key_t o_pms_quality_drive,_says_rcn, 2012.

[13] J. Rankin, C. McGuire, L. Matthews, and D. Ray, "Insight into the increased supervisory role of Senior Charge Nurses: an exploratory study," University of the West of Scotland, Hamilton, 2015.

[14] Royal College of Nursing, "Making the business case for ward sisters/team leader to be supervisory to practice," Retrieved from: http://www.rcn.org.uk/_data/assets/pdf_file/0005/414536/00418 8.pdf, 2011.

[15] K. Evans, "Review of concerns (complaints) handling within NHS Wales - 'Using the gift of complaints," Retrieved from: http://wales.gov.uk/docs/dhss/publications/140702complaintsen.p df, 2014.

[16] C. Agnew, R. Flin, and J.Reid, "Nurse leadership and patient safety," British Medical Journal, vol. 345, pp. e4589, 2012.

[17] G.M. Fealy, M.S. McNamara, M. Casey, R. Geraghty, and M. Butler, et al, "Barriers to clinical leadership development: findings from a national survey," Journal of Clinical Nursing, vol. 20(13 - 14), pp. 2023-2032, 2011.

[18] R.O. Sherman, R. Schwarzkopf, and A.J. Kiger, "Charge nurse perspectives on frontline leadership in acute care environments," International Scholarly Research Notices, E-pub DOI: 10.5402/2011/164052, 2011.

[19] A.M. McCallin, and C. Frankson, "The role of the charge nurse manager: a descriptive exploratory study," Journal of Nursing Management, vol. 18(3), pp. 319-325, 2010.

[20] J.F. Platt, and D. Foster, "Revitalizing the charge nurse role through a bespoke development programme," Journal of Nursing Management, vol. 16(7), pp. 853-857, 2008

[21] H.K. Spence Laschiner, A.C Wong, L.A. Grau, E.A. Read, and L.M. Pineau Stam, "The influence of leadership practices and empowerment on Canadian nurse manager outcomes," Journal of Nursing Management, vol. 20(7), pp. 877-888, 2011.

\section{AUTHORS' PROFILE}

Dr. Jean Rankin, is a Professor in the School of Maternal, Child and Family Health in the University of the West of Scotland, UK. Dr. Jen Rankin was a Leader for the Research and Evaluation.

Clare McGuire is a Registered Nurse and Specialist Community Public Health Nurse (Health Visitor). As manager of the 'Leading Better Care' programme of work; enhancing clinical leadership and supporting the delivery of safe, effective and person centred care have been key priority areas. Clare's professional interests also include; quality improvement; values and competency based recruitment; and cultural competence. Clare is now working in an academic lecturing post at University of the West of Scotland, UK

Dr. Lynsay Matthews is a post-doctoral researcher currently working as a research associate within the University of Glasgow's MRC/CSO Social and Public Health Sciences Unit. Lynsay previously worked as Research Fellow on the Leading Better Care study, exploring the development of leadership skills in senior nursing staff. In addition to health service research her main research interests include physical activity for health and lifestyle behaviour change.

Margot Russell is the Director of NMAHP Practice Development in NHS Lanarkshire and has been a Registered Nurse for 23 years, working in both health care and higher education. Margot's professional experience includes supporting and developing those in professional leadership positions to ensure the delivery of safe, effective and person centred care. Examples of these achievements are through; the development of formal and informal educational programmes; coaching skills; and designing and supporting local initiatives which support leadership in practice.

Devashish Ray is a qualified paediatrician with a current interest in public health research. He has held several research positions within the University of the West of Scotland, including Research Assistant on the Leading Better Care study. He recently completed a Master of Science in Health Studies. 